Results: In all machine learning methods, the accuracy and the area under the receiver operating characteristic (AUROC) were 57.2\% 74.5\%, 0.547 0.747, respectively (Table 1). The accuracy and AUROC of each biologics were similar between machine learning methods. Figure 2 showed interpretation of feature importance with the Shapley plot for remission. The most important feature was age in adalimumab (younger were closer to remission), daily corticosteroid dose in etanercept, golimumab, and all TNF inhibitors (using fewer doses daily were closer to remission), baseline erythrocyte sedimentation rate in infliximab (lower ESR were closer to remission), disease duration in abatacept (longer disease durations showed difficulty determining remission), baseline c-reactive protein in tocilizumab (higher CRP were closer to remission).

Table 1. Predicting remission for all biologics in various machine learning method.

\begin{tabular}{llllllllll}
\hline & Measure & Lasso & Ridge & SVM & $\begin{array}{c}\text { Random } \\
\text { Forest }\end{array}$ & XGBoost & No info rate & Sample \\
\hline Abatacept & Accuracy & $74.1 \%$ & $74.1 \%$ & $70.6 \%$ & $71.8 \%$ & $68.8 \%$ & $70.6 \%$ & 216 \\
& AUROC & 0.725 & 0.742 & 0.707 & 0.677 & 0.647 & 0.500 & \\
Adalimumab & Accuracy & $73.6 \%$ & $72.0 \%$ & $70.4 \%$ & $72.0 \%$ & $70.4 \%$ & $68.8 \%$ & 315 \\
& AUROC & 0.710 & 0.729 & 0.700 & 0.675 & 0.663 & 0.500 & \\
Etanercept & Accuracy & $72.0 \%$ & $72.0 \%$ & $70.0 \%$ & $71.5 \%$ & $70.0 \%$ & $68.0 \%$ & 250 \\
& AUROC & 0.741 & 0.747 & 0.726 & 0.719 & 0.704 & 0.500 & \\
Golimumab & Accuracy & $71.3 \%$ & $68.5 \%$ & $66.7 \%$ & $68.5 \%$ & $68.5 \%$ & $68.5 \%$ & 138 \\
& AUROC & 0.746 & 0.727 & 0.701 & 0.690 & 0.655 & 0.500 & \\
TNFiximab inhibitors & Accuracy & $72.8 \%$ & $73.5 \%$ & $67.6 \%$ & $73.5 \%$ & $69.1 \%$ & $72.5 \%$ & 172 \\
& AUROC & 0.663 & 0.683 & 0.616 & 0.597 & 0.527 & 0.500 & \\
Tocilizumab & Accuracy & $73.9 \%$ & $74.5 \%$ & $73.9 \%$ & $74.2 \%$ & $73.6 \%$ & $70.3 \%$ & 875 \\
& AUROC & 0.739 & 0.741 & 0.726 & 0.747 & 0.724 & 0.500 & \\
& Accuracy & $62.4 \%$ & $63.6 \%$ & $62.4 \%$ & $59.5 \%$ & $57.2 \%$ & $59.5 \%$ & 436 \\
& AUROC & 0.633 & 0.640 & 0.633 & 0.615 & 0.547 & 0.500 & \\
\hline
\end{tabular}
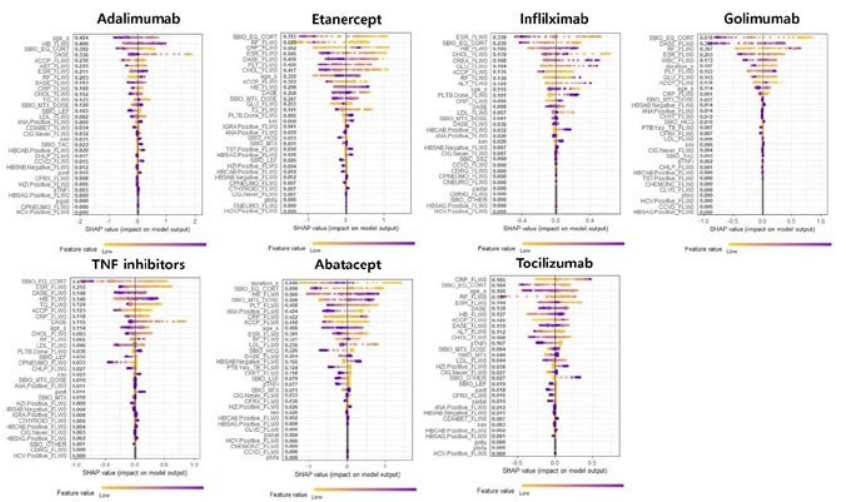

Figure 2. Shapley plots and SHAP values for the feature importance from clinical information in patients with RA.

Conclusion: We developed machine learning models for predicting remission as a response to each biologics in active RA patients based on their clinical profiles, and found important clinical features using explainable Al. This approach may support clinical decisions to improve treatment outcomes in patients with RA.

Disclosure of Interests: None declared

DOI: 10.1136/annrheumdis-2020-eular.3438

\section{OP0024 USE OF HYDROXYCHLOROQUINE AND RISK OF HEART FAILURE IN PATIENTS WITH RHEUMATOID ARTHRITIS}

A. Sorour ${ }^{1}$, Y. Shahin ${ }^{1}$, C. S. Crowson ${ }^{2}$, R. Kurmann ${ }^{1}$, S. Achenbach ${ }^{2}$, R. Mankad ${ }^{1}$, E. Myasoedova ${ }^{3} .{ }^{1}$ Mayo Clinic, Cardiovascular diseases, Rochester, United States of America; ${ }^{2}$ Mayo Clinic, Biomedical Statistics and Informatics, Rochester, United States of America; ${ }^{3}$ Mayo Clinic, Rheumatology, Rochester, United States of America

Background: Hydroxychloroquine ( $\mathrm{HCQ}$ ) is a disease-modifying anti-rheumatic drug (DMARD) used as a long-term treatment for rheumatoid arthritis (RA) patients. Cardiotoxicity is a rare but potentially life-threatening side effect of $\mathrm{HCQ}$ and may present as conduction disorders, cardiomyopathy, and resulting heart failure (HF). The evidence of cardiotoxicity associated with the use of HCQ largely relies on case reports and case series while large cohort studies on the subject are lacking.

Objectives: To examine the relationship between the use of $\mathrm{HCQ}$ and risk of developing HF in RA.

Methods: In this nested case-control study, cases were Olmsted county, Minnesota residents with incident RA (based on 1987 ACR criteria) in 1980-2013 who developed HF after RA incidence. Each case was matched on year of birth, sex and year of RA incidence with an RA control who did not develop HF. Each non-HF control was assigned an index date corresponding to the HF diagnosis date of the case. Controls were allowed to later become cases to avoid bias. HF was defined using the Framingham criteria. Data on HCQ use including start and stop dates and dose changes was manually abstracted via medical record review, and used to calculate HCQ duration and cumulative dose. Age-adjusted logistic regression models were used to examine the association between HCQ and HF. Results: From a cohort of 1078 subjects, the study identified 143 RA cases diagnosed with HF (mean age 65.8, 62\% females) and 143 non-HF RA controls (mean age 64.5 $62 \%$ female). Cases and controls had similar RA duration, proportion of patients positive for rheumatoid factor (RF) and/ or cyclic citrullinated antibody (CCP), body mass index, and smoking status (Table). The duration of HCQ use prior to the diagnosis of HF was 2.8 years in cases and 2.6 years in controls. A total of 71 cases and 69 controls used $\mathrm{HCQ}$ at some time before index date. Among these, the median (interquartile range) duration of $\mathrm{HCQ}$ use was $2.8(0.6,10.0)$ years for cases and $2.5(0.7,8.2)$ for controls The median cumulative dose of $\mathrm{HCQ}$ was $371 \mathrm{~g}$ and $302 \mathrm{~g}$ in cases and controls, respectively, with $55 \%$ of cases receiving a cumulative dose of $\geq 300 \mathrm{~g}$ compared to $54 \%$ in controls. $\mathrm{HCQ}$ cumulative dose was not associated with $\mathrm{HF}$ (Odds Ratio [OR]: 0.96 per $100 \mathrm{~g}$ increase in cumulative dose, 95\% confidence interval [95\% Cl]: 0.90-1.03). Likewise, no association was found for patients with a cumulative dose $\geq 300 \mathrm{~g}$ (OR $0.92,95 \% \mathrm{Cl} 0.41$ 2.08). The duration of use of HCQ prior to index was not associated with HF (OR 0.98 $95 \% \mathrm{Cl} 0.91-1.05)$. Retinal toxicity rates were similar in cases and controls.

Table. Characteristics of patients with rheumatoid arthritis with and without heart failure.

\begin{tabular}{lcc}
\hline Variable & $\mathrm{HF}$ & non-HF \\
\hline Age at RA diagnosis (years) & $65.8 \pm 12.3$ & $64.5 \pm 12.5$ \\
Female & $62 \%$ & $62 \%$ \\
RA duration at baseline (years) & $11.3 \pm 8.5$ & $10.3 \pm 8.2$ \\
RF positive & $66 \%$ & $65 \%$ \\
CCP positive & $46 \%$ & $53 \%$ \\
RF/ CCP positive & $68 \%$ & $66 \%$ \\
BMI (at RA diagnosis) & $28.6 \pm 6.5$ & $27.7 \pm 5.4$ \\
Smoking status at RA incidence & $45 \%$ & $41 \%$ \\
Former & $22 \%$ & $22 \%$ \\
Current & & \\
\hline
\end{tabular}

Conclusion: Use of HCQ was not associated with development of HF in patients with RA in this study. While there was no statistically significant association between the cumulative dose of $\mathrm{HCQ}$ and $\mathrm{HF}$, the confidence interval for $\mathrm{HCQ}$ dose $\geq 300 \mathrm{~g}$ was wide suggesting that more studies are needed to understand the impact of higher doses of HCQ on development of HF in RA.

Disclosure of Interests: Ahmed Sorour: None declared, Youssef Shahin: None declared, Cynthia S. Crowson Grant/research support from: Pfizer research grant, Reto Kurmann: None declared, Sara Achenbach: None declared, Rekha Mankad: None declared, Elena Myasoedova: None declared DOI: 10.1136/annrheumdis-2020-eular.625

\section{OP0025 DRUG RETENTION OF 7 BIOLOGICS AND TOFACITINIB IN BIOLOGICS-NAÏVE AND BIOLOGICS-SWITCHED PATIENTS WITH RHEUMATOID ARTHRITIS -THE ANSWER COHORT STUDY}

K. Ebina ${ }^{1}$, T. Hirano ${ }^{2}$, Y. Maeda ${ }^{2}$, W. Yamamoto ${ }^{3,4}$, M. Hashimoto ${ }^{4}$, K. Murata ${ }^{4}$, T. Takeuchi ${ }^{5}$, H. Shiba ${ }^{5}$, Y. Son ${ }^{6}$, H. Amuro ${ }^{6}$, A. Onishi ${ }^{7}$, K. Akashi ${ }^{7}$, R. Hara ${ }^{8}$, M. Katayama ${ }^{9}$, K. Yamamoto ${ }^{10}$, A. Kumanogoh ${ }^{2}$, M. Hirao ${ }^{11} .{ }^{1}$ Osaka University, Graduate School of Medicine, Department of Musculoskeletal Regenerative Medicine, Osaka, Japan; ${ }^{2}$ Osaka University, Graduate School of Medicine, Department of Respiratory Medicine and Clinical Immunology, Osaka, Japan; ${ }^{3}$ Kurashiki Sweet Hospital, Department of Health Information Management, Okayama, Japan; ${ }^{4}$ Graduate School of Medicine, Kyoto University, Department of Advanced Medicine for Rheumatic Diseases, Kyoto, Japan; ${ }^{5}$ Osaka Medical College, Department of Internal Medicine (4), Osaka, Japan; ${ }^{6}$ Kansai Medical University, First Department of Internal Medicine, Osaka, Japan; ${ }^{7}$ Kobe University Graduate School of Medicine, Department of Rheumatology and Clinical Immunology, Hyogo, Japan; ${ }^{8}$ Nara Medical University, The Center for Rheumatic Diseases, Nara, Japan; ${ }^{9}$ Osaka Red Cross Hospital, Department of Rheumatology, Osaka, Japan; ${ }^{10}$ Wakayama Medical University Hospital, Department of Medical Informatics, Wakayama, Japan; ${ }^{11}$ Osaka University, Graduate School of Medicine, Department of Orthopaedic Surgery, Osaka, Japan

Background: EULAR recommendation announced that biological disease-modifying antirheumatic drugs (bDMARDs) and janus kinase inhibitors (JAKi) are considered as equivalent in the treatment of rheumatoid arthritis (RA). However we still lack reliable evidence of direct comparison between these agents' retention, which may reflect both effectiveness and safety. 